\title{
Población anciana y campañas para la prevención de la hipercolesterolemia en Salamanca
}

\author{
J. I. HERRERO HERRERO, J. A. MARTÍN OTERINO, F. SANZ ORTEGA, A. \\ MATEOS SÁNCHEZ, J. M. POLO GARCÍA, M. L. GARCÍA GÓMEZ, F. PASCUAL \\ GONZÁLEZ
}

Unidad de Lípidos. Complejo Hospitalario de Salamanca. Salamanca

\author{
THE ELDERLY AND CAMPAIGNS FOR THE PREVENTION OF \\ HYPERCHOLESTEROLEMIA IN SALAMANCA (SPAIN)
}

\begin{abstract}
RESUMEN
Objetivo: Analizar el perfil de riesgo cardiovascular de los individuos mayores de 65 años asistentes a distintas "Jornadas sobre colesterol y aterosclerosis" realizadas, durante 1999 , en diferentes poblaciones de la provincia de Salamanca.

Material y métodos: Durante su asistencia a las jornadas, los voluntarios de cualquier edad fueron sometidos a un cuestionario y a distintas mediciones clínico-analíticas.

Resultados: Asistieron a las jornadas 267 voluntarios $(40,8 \%$ de ellos varones) mayores de 65 años. Los valores medios de los parámetros estudiados fueron: edad $=72,3 \pm 6,5$ años; índice de masa corporal (IMC)= $26,8 \pm 4,5 \mathrm{~kg} / \mathrm{m}^{2}$; presión arterial sistólica $(\mathrm{PAS})=139,8 \pm 20,6 \mathrm{mmHg}$; presión arterial diastólica $(\mathrm{PAD})=80,0 \pm 10,1 \mathrm{mmHg}$; y colesterol total $(\mathrm{CT})=226,2 \pm 44,9 \mathrm{mg} / \mathrm{dl}$. El porcentaje de tabaquismo fue del $5,2 \%$. Las cifras de PAS mostraron correlación significativa positiva con la edad $(p<0,05)$ y con la PAD $(p<0,01)$. En el subgrupo de los varones, la edad evolucionó inversamente a las cifras de CT $(\mathrm{p}<0,01)$. Globalmente, 203 voluntarios $(76,0 \%)$ tuvieron cifras de CT $\geq 200 \mathrm{mg} / \mathrm{dl} ; 78(29,2 \%) \geq 250$ $\mathrm{mg} / \mathrm{dl}$; y $12(4,5 \%)$ concentraciones $\geq 300 \mathrm{mg} / \mathrm{dl}$. Detectamos presiones arteriales con cifras de PAS $\geq 140 \mathrm{mmHg}$ en $143(53,6 \%)$ individuos; PAS $\geq 180 \mathrm{mmHg}$ en $11(4,1 \%)$; PAD $\geq 90 \mathrm{mmHg}$ en $72(27,0 \%)$; y PAD $\geq 110 \mathrm{mmHg}$ en $4(1,5 \%)$. El IMC fue $\geq 30 \mathrm{~kg} / \mathrm{m}^{2}$ en $54(20,2 \%)$ personas del estudio. El 90,8\% de los voluntarios con enfermedad cardiovascular previa eran portadores, al menos, de otro factor de riesgo cardiovascular no controlado; el 24,1\% de al menos dos factores de riesgo; y el 9,1\% de al menos tres. En 38,5\% ocasiones mostraron niveles elevados de CT y de PAS, simultáneamente.

Conclusiones: Nuestros datos, así como las evidencias actuales sobre el beneficio del control de factores de riesgo cardiovascular en los ancianos, sugieren la necesidad de establecer estrategias para un mejor control del riesgo cardiovascular en este grupo etario de población en nuestra provincia.
\end{abstract}

PALABRAS CLAVE:Ancianos. Enfermedades cardiovasculares. Factores de riesgo. Epidemiología. Colesterol. Obesidad. Tabaquismo.

\section{ABSTRACT}

Objective: To analyze the cardiovascular risk profile of the persons older than 65 years of age who participated in different "Days on Cho lesterol and Atherosclerosis" organized during 1999 in different towns of the province of Salamanca.

Material and methods: The volunteers of any age were subjected to a questionnaire and to several clinical and laboratory measurements.

Results: The age of 267 volunteers ( $40.8 \%$ males) attending the cam paign was $\geq 65$ years. The mean values of the studied parameters were: age $=72.3 \pm 6.5$ years; body mass index $(B M I)=26.8 \pm 4.5 \mathrm{~kg} / \mathrm{m}$; systolic blood pressure $(S B P)=139.8 \pm 20.6 \mathrm{mmHg}$; dyastolic blood pressure $(D B P)=80.0 \pm 10.1 \mathrm{mmHg} ;$ and total cholesterol $(T C)=226.2 \pm 44.9$ $\mathrm{mg} / \mathrm{dl}$. The percentage of smoking habit was $5.2 \%$. The figures of SBP showed positive significant correlation with those of age $(p<0.05)$ and $\operatorname{DBP}(p<0.01)$. Among males, the age evolved inversely to the numbers of TC $(p<0.01)$. Globally, 203 volunteers $(76.0 \%)$ had concentrations of $T C \geq 200 \mathrm{mg} / \mathrm{dl}$; $78(29.2 \%) \geq 250 \mathrm{mg} / \mathrm{dl}$; and $12(4.5 \%)$ concentra tions $\geq 300 \mathrm{mg} / \mathrm{dl}$. We detected blood pressure levels of $S B P \geq 140 \mathrm{mmHg}$ in $143(53.6 \%)$ individuals; $S B P \geq 180 \mathrm{mmHg}$ in 11 (4.1\%); DBP $\geq 90$ $\mathrm{mmHg}$ in 72 (27.0\%); and $D B P \geq 110 \mathrm{mmHg}$ in $4(1.5 \%)$. The BMI was $\geq 30 \mathrm{~kg} / \mathrm{m}^{2}$ in $54(20.2 \%)$ people of the study. $90.8 \%$ of the volunteers with previous cardiovascular disease carried, at least, another not con trolled cardiovascular risk factor; $24.1 \%$ had at least two risk factors; and $9.1 \%$ had, at least, three. In 38.5\% they showed high levels of TC and DBP, simultaneously.

Conclusions: Our data, together with the current evidences about the benefit of the control of cardiovascular risk factors in the elderly, sug gest the necessity to establish strategies for a better control of the car diovascular risk in this group of age in our province.

KEY WORDS: Elderly. Cardiovascular diseases. Risk factors. Epide miology. Cholesterol. Obesity. Smoking.

Herrero Herrero JI, Martín Oterino JA, Sanz Ortega F, Mateos Sánchez A, Polo García JM, García Gómez ML, Pascual González F. Población anciana y campañas para la prevención de la hipercolesterolemia en Salamcanca. An Med Interna (Madrid) 2001; $18: 13-19$.

\section{INTRODUCCIÓN}

El acontecimiento demográfico más relevante en la sociedad occidental actual es el rápido envejecimiento de la población. La población española mayor de 65 años, proyectada a 1 de julio de 2000, se ha cifrado en 6.642.200 habitantes, que representa el 16,8\% del total (1). Este porcentaje superaba, ya en 1996, el 20\% entre los habitantes de Castilla y León (2). En estas personas (que, siguiendo el criterio tradicional, denominamos ancianos) las enfermedades cardiovasculares represen-

Trabajo aceptado: 20 de Julio de 2000

Correspondencia: J. Ignacio Herrero Herrero. Servicio de Medicina Interna. Hospital Virgen del Castañar. Travesía de Santa Ana, nº 4 - 37700 Béjar. Salamanca. 
tan la primera causa de muerte (1) y el origen de una elevada morbilidad con repercusiones económico/sanitarias en España y en el mundo occidental. Todo ello y la reciente evidencia de los beneficios de la intervención farmacológica sobre los niveles séricos de colesterol en la prevención primaria $(3,4)$ y secundaria $(5,6)$ de la enfermedad cardiovascular en individuos incluidos en este grupo de edad, ha renovado el interés por el control de este factor de riesgo en los ancianos.

Durante el primer semestre de 1999, organizamos en distintas localidades de la provincia de Salamanca unas "Jornadas sobre colesterol y aterosclerosis", dirigidas a la detección de hipercolesterolemia y otros factores de riesgo cardiovascular entre la población, y a la información sobre distintos aspectos de las enfermedades cardiovasculares. El análisis de los datos recogidos entre los participantes en las mismas mayores de 65 años constituye el objetivo de este estudio.

\section{MATERIAL Y MÉTODOS}

El desarrollo y contenido de las "Jornadas sobre colesterol aterosclerosis" ha sido comunicado previamente (7). En breve, estas tuvieron lugar en las localidades de Salamanca, Ciudad Rodrigo y Béjar. Convocadas a través de los medios de comunicación locales y dirigidas a toda la población, durante las mismas los voluntarios asistentes fueron sometidos a un cuestionario y a mediciones que incluían datos sobre los siguientes parámetros: nombre, edad (años cumplidos), sexo, antecedentes personales de enfermedad cardiovascular (enfermedad coronaria, enfermedad arterial periférica, ictus/AIT), antecedentes familiares de enfermedad cardiovascular (idem), antecedentes personales de tabaquismo (se interrogaba sobre si, en el momento de la encuesta, eran fumadores habituales), peso $(\mathrm{kg})$, talla $(\mathrm{m})$, presión arterial $(\mathrm{mm} \mathrm{Hg}$; medida por la técnica indirecta estándar utilizando un manómetro de mercu- rio), y colesterol total ( $\mathrm{mg} / \mathrm{dl}$; cuantificado en sangre capilar mediante un Reflotron ${ }^{\circledR}$, homologado).

En la tabulación de los datos, los referentes a los antecedentes familiares, personales y al hábito tabáquico se expresaron como variables cualitativas dicotómicas (sí/no). Las variables peso y talla se combinaron en el parámetro "índice de masa corporal" $\left(\mathrm{IMC}=\right.$ peso $[\mathrm{kg}] /$ talla $\left.[\mathrm{m}]^{2}\right)$. La comparación de las variables cuantitativas en los distintos grupos se realizó mediante el estadístico t de Student. Empleamos el test $C h i^{2}$ (con la corrección de Yates o sustituido por el test exacto de Fisher cuando las condiciones de aplicación lo requerían) para comparar frecuencias. El coeficiente $\rho_{x, y}=\operatorname{COV}(\mathrm{x}, \mathrm{y}) /\left(\sigma_{\mathrm{x}} \cdot \sigma_{\mathrm{y}}\right)$ fue utilizado para resolver los problemas de correlación (Microsoft ${ }^{\circledR}$ Excel 97). En todos los casos se estableció un riesgo $\alpha=0,05$ como umbral de significación estadística.

\section{RESULTADOS}

Participaron en las jornadas 267 (109 varones [40,8\%] y 158 mujeres [59,2\%]) voluntarios mayores de 65 años, que representaron el 49,4\% de las 541 personas de cualquier edad asistentes a las mismas en las que la disponibilidad de tiempo y material hizo posible la cumplimentación de los cuestionarios y la recogida y análisis de las muestras. La distribución de los datos según la procedencia, sexo y grupos de edad, así como su evaluación estadística, se recoge en las tablas I, II y III.

El análisis de la matriz de correlación de los parámetros cuantitativos incluidos en el estudio únicamente demostró valores significativos, positivos, entre las cifras de presión arterial sistólica (PAS) respecto a los de la edad $(\mathrm{p}<0,05)$ y la presión arterial diastólica (PAD) $(\mathrm{p}<0,01)$. Por sexos, mientras que en el subgrupo de los varones se demostró una correlación negativa entre edad y colesterol total (CT) $(\mathrm{p}<0,01)$,

TABLA I

DATOS GLOBALES Y DISTRIBUIDOS POR LOCALIDADES DE PRO CEDENCIA DE LOS ANCIANOS PARTICIPANTES EN EL ESTUDIO

\begin{tabular}{lllll}
\hline & Total & Salamanca & Béjar & C. Rodrigo \\
\hline No de participantes & 267 & 92 & 65 & 110 \\
Sexo: varón $(\mathrm{n}(\%))$ & $109(40,8 \%)$ & $51(55,4 \%)^{\mathrm{a}}$ & $23(35,4 \%)$ & $35(31,8 \%)$ \\
mujer $(\mathrm{n}(\%))$ & $158(59,2 \%)$ & $41(44,6 \%)$ & $42(64,6 \%)$ & $75(68,2 \%)$ \\
Edad (años) & $72,3 \pm 6,5$ & $70,0 \pm 4,8^{\mathrm{b}}$ & $72,1 \pm 5,4^{\mathrm{c}}$ & $74,3 \pm 7,6$ \\
IMC (kg/m) & $26,8 \pm 4,5$ & $26,6 \pm 4,8$ & $26,8 \pm 4,9$ & $26,9 \pm 4,0$ \\
PAS (mm Hg) & $139,8 \pm 20,6$ & $139,2 \pm 20,7$ & $136,2 \pm 20,0$ & $142,5 \pm 20,8$ \\
PAD (mm Hg) & $80,0 \pm 10,1$ & $81,7 \pm 8,8^{\mathrm{d}}$ & $77,2 \pm 10,3$ & $80,3 \pm 10,7$ \\
CT (mg/dl) & $226,2 \pm 44,9$ & $222,4 \pm 52,5$ & $232,7 \pm 36,2$ & $225,5 \pm 42,6$ \\
AF (n (\%)) & $95(35,6 \%)$ & $56(60,9 \%)^{\mathrm{e}}$ & $15(23,1 \%)$ & $24(21,8 \%)$ \\
AP (n(\%)) & $65(24,3 \%)$ & $49(53,3 \%)^{\mathrm{f}}$ & $6(9,2 \%)$ & $10(9,1 \%)$ \\
Tabaquismo (n (\%)) & $14(5,2 \%)$ & $7(7,6 \%)$ & $3(4,6 \%)$ & $4(3,6 \%)$ \\
\hline
\end{tabular}

IM C: índice de masa corporal; PAS: presión arterial sistólica; PAD: presión arterial diastólica; CT: colesterol total; AF: antecedentes familiares de enfermedad cardiovascular; AP: antecedentes personales de enfermedad cardiovascular.

a Diferencia estadísticamente significativa $(p<0,05)$ respecto a la proporción en Ciudad Rodrigo. ${ }^{\mathrm{b}}$ Diferencia estadísticamente significativa respecto a los resultados en Béjar $(p<0,05)$ y en Ciudad Rodrigo $(p<0,00001)$. ' Diferencia estadísticamente significativa respecto a los resultados en Ciudad Rodrigo $(p<0,05)$. 'Diferencia estadísticamente significativa respecto a los resultados en Béjar $(p<0,01)$. e Diferencia estadísticamente significativa respecto a las proporciones en Béjar $(p<0,01)$ y en Ciudad Rodrigo $(p<0,001) .{ }^{f}$ Diferencia estadísticamente significativa respecto a las proporciones en Béjar $(p<0,0001)$ y en Ciudad Rodrigo $(p<0,00001)$. 
TABLA ॥

DISTRIBUCION DE LOS RESULTADOS POR SEXO

\begin{tabular}{lll}
\hline & Varones & \multicolumn{1}{c}{ M ujeres } \\
\hline № de participantes & 109 & 158 \\
Edad (años) & $72,3 \pm 6,5$ & $72,3 \pm 6,5$ \\
IM C (kg/ m²) & $26,7 \pm 4,8$ & $26,8 \pm 4,3$ \\
PAS (mm Hg) & $139,9 \pm 30,0$ & $139,8 \pm 18,1$ \\
PAD (mm Hg) & $80,2 \pm 10,2$ & $79,9 \pm 10,0$ \\
CT (mg/dl) & $209,9 \pm 48,6^{a}$ & $237,4 \pm 38,4$ \\
AF $(\mathrm{n}(\%))$ & $39(35,8 \%)$ & $56(35,4 \%)$ \\
AP (n (\% ) & $31(28,4 \%)$ & $34(21,5 \%)$ \\
Tabaquismo (n $(\%))$ & $11(10,1 \%)^{b}$ & $3(1,9 \%)$
\end{tabular}

IMC: índice de masa corporal; PAS: presión arterial sistólica; PAD: presión arterial diastólica; CT: colesterol total; AF: antecedentes familiares de enfermedad cardiovascular; AP: antecedentes personales de enfermedad cardiovascular.

Diferencias estadísticamente significativas respecto a los resultados en los varones: a $p<0,000001 ;{ }^{b} p<0,01$.

este hallazgo no fue verificado entre las mujeres. Por grupos de edad, se observó una correlación positiva entre el IMC $(\mathrm{p}<0,01)$ y la TAD $(\mathrm{p}<0,01)$ sólo a partir de los 71 años. En el grupo "71 - 75 años" se detectaron, además, correlaciones negativas significativas entre las variables: edad/PAD $(\mathrm{p}<0,01)$ y edad/CT $(\mathrm{p}<0,05)$; y positivas entre PAS/CT $(\mathrm{p}<0,05)$.

Globalmente, 203 voluntarios $(76,0 \%)$ tuvieron cifras de CT $\geq 200 \mathrm{mg} / \mathrm{dl} ; 78(29,2 \%) \geq 250 \mathrm{mg} / \mathrm{dl} ;$ y $12(4,5 \%)$ concentraciones $\geq 300 \mathrm{mg} / \mathrm{dl}$. Detectamos presiones arteriales con cifras de PAS $\geq 140 \mathrm{~mm} \mathrm{Hg}$ en 143 (53,6\%) individuos; PAS $\geq 180 \mathrm{~mm} \mathrm{Hg}$ en $11(4,1 \%) ; \mathrm{PAD} \geq 90 \mathrm{~mm} \mathrm{Hg}$ en $72(27,0 \%)$; y PAD $\geq 110 \mathrm{~mm} \mathrm{Hg}$ en $4(1,5 \%)$. El IMC fue $\geq 30 \mathrm{~kg} / \mathrm{m}^{2}$ en 54 $(20,2 \%)$ personas del estudio. No se observaron diferencias por sexos en estas reparticiones (tabla IV).

El grupo de ancianos voluntarios que referían antece- dentes personales de enfermedad cardiovascular mostró una edad media (70,8 $\pm 5,5$ años) inferior a la de aquéllos que carecían de ellos $(72,7 \pm 5,5$ años; $p<0,05)$, así como un mayor porcentaje de antecedentes familiares positivos $(78,5 \%$ frente a $21,8 \% ; \mathrm{p}<0,00001)$. No se observaron diferencias entre estos grupos en el resto de los parámetros analizados (tabla V).

Los voluntarios con enfermedad cardiovascular previa presentaron cifras de PAS $\geq 140 \mathrm{~mm} \mathrm{Hg}$ en el $50,8 \%$ de los casos y de CT $\geq 200 \mathrm{mg} / \mathrm{dl}$ en el $75,4 \%$. La incidencia de otros factores de riesgo modificables en este grupo se recoge en la tabla VI. Cincuenta y nueve de ellos $(90,8 \%)$ eran portadores, al menos, de un factor de riesgo cardiovascular no controlado; $37(24,1 \%)$ de al menos dos factores de riesgo; y $14(9,1 \%)$ de al menos 3. En $25(38,5 \%)$ ocasiones los voluntarios con patología cardiovascular previa mostraron niveles elevados de CT y de PAS simultáneamente (entre ellos, 14 presentaban adicionalmente algún otro factor no controlado).

\section{DISCUSIÓN}

Desde la perspectiva de la prevención primaria de la hipercolesterolemia en la población general, la detección oportunista de casos (8) y la puesta en práctica de campañas de prevención han sido recomendadas y utilizadas (9) en nuestro país durante los últimos años.

En las Jornadas de Salamanca, los mayores de 65 años supusieron prácticamente la mitad de los asistentes. Esta circunstancia (evidenciada en otras campañas similares) (9) revela tanto el interés de este sector de la población por los problemas relacionados con la salud, como su disponibilidad, al carecer de obligaciones laborales, para participar en este tipo de actos.

En nuestro estudio, los niveles medios de colesterol (226 \pm $44,9 \mathrm{mg} / \mathrm{dl}$ ) fueron superiores a los comunicados en algunos trabajos nacionales $(10,11)$. Asimismo, nuestro porcentaje de ancianos con CT $\geq 200 \mathrm{mg} / \mathrm{dl}(76,0 \%)$ superó al del estudio de Framingham (71\%) (12), al hallado en el sur de Europa en el

TABLA III

DISTRIBUCION DE LOS RESULTADOS POR GRUPOS DE EDAD

\begin{tabular}{llll}
\hline & $65-70$ años & $71-75$ años & $\geq 76$ años \\
\hline No de participantes & 131 & 72 & 64 \\
Sexo: varón (n (\%)) & $55(42,0 \%)$ & $26(36,1 \%)$ & $28(43,7 \%)$ \\
Edad (años) & $76(58,0 \%)$ & $46(63,9 \%)$ & $36(56,3 \%)$ \\
IM C (kg/m $\left.{ }^{2}\right)$ & $67,3 \pm 1,7$ & $72,7 \pm 1,4$ & $81,9 \pm 5,0$ \\
PAS (mm Hg) & $26,7 \pm 3,9$ & $27,7 \pm 3,9$ & $26,1 \pm 5,9$ \\
PAD (mm Hg) & $137,1 \pm 18,4$ a & $140,2 \pm 25,7$ & $145,0 \pm 17,8$ \\
CT (mg/dl) & $79,9 \pm 10,5$ & $80,7 \pm 10,0$ & $79,5 \pm 9,4$ \\
AF (n (\%)) & $227,4 \pm 48,5$ & $226,5 \pm 38,0$ & $223,4 \pm 45,0$ \\
AP (n (\%)) & $55(42,0 \%)$ & $28(38,9 \%)$ & $12(18,8 \%)^{b}$ \\
Tabaquismo & $37(28,2 \%)$ & $18(25,0 \%)$ & $10(15,6 \%)$ \\
\hline
\end{tabular}

IIM C: índice de masa corporal; PAS: presión arterial sistólica; PAD: presión arterial diastólica; CT: colesterol total; AF: antecedentes familiares de enfermedad cardiovascular; AP: antecedentes personales de enfermedad cardiovascular.

a Diferencia estadísticamente significativa respecto a los resultados en el grupo " $\geq 76$ años" $(p<0,01)$. ${ }^{b}$ Diferencia estadísticamente significativa respecto a los resultados en los grupos " 65 - 70 años" $(p<0,05)$ y " 71 - 75 años" $(p<0,05)$. 
TABLA IV

NIVELES DE FACTO RES DE RIESGO SEGÚN EL SEXO DE LOS INDIVIDUOS ESTUDIADOS

\begin{tabular}{|c|c|c|c|c|}
\hline \multicolumn{2}{|c|}{ № de participantes } & \multirow{2}{*}{ 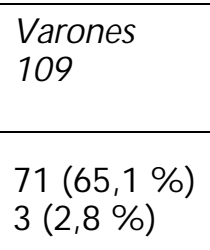 } & \multirow{2}{*}{$\begin{array}{l}\text { M ujeres } \\
158 \\
132(83,5 \%) \\
9(5,7 \%)\end{array}$} & \multirow{2}{*}{$\begin{array}{l}\mathrm{p} \\
\text { NS } \\
\text { NS }\end{array}$} \\
\hline $\mathrm{CT}$ & $\begin{array}{l}\geq 200 \mathrm{mg} / \mathrm{dl}(\mathrm{n}(\%)) \\
\geq 300 \mathrm{mg} / \mathrm{dl}(\mathrm{n}(\%))\end{array}$ & & & \\
\hline PAS & $\begin{array}{l}\geq 140 \mathrm{~mm} \mathrm{Hg}(\mathrm{n}(\%)) \\
\geq 180 \mathrm{~mm} \mathrm{Hg}(\mathrm{n}(\%))\end{array}$ & $\begin{array}{l}58(53,2 \%) \\
4(3,7 \%)\end{array}$ & $\begin{array}{l}85(53,8 \%) \\
7(4,4, \%)\end{array}$ & $\begin{array}{l}\text { NS } \\
\text { NS }\end{array}$ \\
\hline PAD & $\begin{array}{l}\geq 90 \mathrm{~mm} \mathrm{Hg}(\mathrm{n}(\%)) \\
\geq 110 \mathrm{~mm} \mathrm{Hg}(\mathrm{n}(\%))\end{array}$ & $\begin{array}{l}32(29,6 \%) \\
2(1,8 \%)\end{array}$ & $\begin{array}{l}40(57,0 \%) \\
2(1,3 \%)\end{array}$ & $\begin{array}{l}\text { NS } \\
\text { NS }\end{array}$ \\
\hline IMC & $\geq 30 \mathrm{~kg} / \mathrm{m}^{2}(\mathrm{n}(\%))$ & $22(20,2 \%)$ & $32(20,3 \%)$ & NS \\
\hline
\end{tabular}

CT: colesterol total; PAS: presión arterial sistólica; PAD: presión arterial diastólica; IM C: índice de masa corporal; NS: no significativo (valor de p>0,05).

TABLA V

CARACTERÍSTICAS DE LOS INDIVIDUOS DEL ESTUDIO SEGÚN TUVIERAN O NO ANTECEDENTES PERSO NALES DE ENFERM EDAD CARDIOVASCULAR

\begin{tabular}{|c|c|c|c|}
\hline № de participantes & $\begin{array}{l}\text { Con antecedentes } \\
65\end{array}$ & $\begin{array}{l}\text { Sin antecedentes } \\
202\end{array}$ & $p$ \\
\hline $\begin{array}{ll}\text { Sexo: } & \text { varón }(\mathrm{n}(\%)) \\
\text { muier }(\mathrm{n}(\%))\end{array}$ & $31(47,7 \%)$ & $78(38,6 \%)$ & NS \\
\hline Edad (años) & $70,8 \pm 5,5$ & $\begin{array}{l}124(61,4 \%) \\
72,7 \pm 6,7\end{array}$ & $<0,05$ \\
\hline IM C $\left(\mathrm{kg} / \mathrm{m}^{2}\right)$ & $27,5 \pm 3,8$ & $26,6 \pm 4,7$ & NS \\
\hline PAS $(\mathrm{mm} \mathrm{Hg})$ & $139,2 \pm 17,6$ & $140,0 \pm 21,6$ & NS \\
\hline PAD (mm Ha) & $80,3 \pm 9,9$ & $79,910,2$ & NS \\
\hline $\mathrm{CT}(\mathrm{mg} / \mathrm{dl})$ & $220,640,5$ & $228,0 \pm 46,2$ & NS \\
\hline $\mathrm{AF}(\mathrm{n}(\%))$ & $51(78,5 \%)$ & $44(21,8 \%)$ & $<0,000001$ \\
\hline Tabaquismo (n (\%)) & $3(4,3 \%)$ & $11(5,4 \%)$ & NS \\
\hline
\end{tabular}

IM C: índice de masa corporal; PAS: presión arterial sistólica; PAD: presión arterial diastólica; CT: colesterol total; AF: antecedentes familiares de enfermedad cardiovascular; NS: no significativo (valor de $p>0,05$ ).

proyecto WHO-ERICA (26\% con CT > $250 \mathrm{mg} / \mathrm{dl} ; 29,2 \%$ en nuestra serie) (13) y al más reciente Cardiovascular Health Study $(61 \%)$ (14). Como en otros trabajos $(9,10,15,16)$ las cifras de CT fueron más altas en las mujeres, siendo significativa la observación de una correlación negativa entre la edad y las cifras de CT sólo en el caso de los varones (al contrario de lo objetivado en un estudio europeo sobre 5310 hipertensos mayores de 60 años, en el que este descenso se observó sólo en el caso de las mujeres) (17).

Respecto a la presión arterial, el porcentaje de pacientes hipertensos de nuestro estudio (PAS $\geq 140 \mathrm{~mm} \mathrm{Hg}$ y/o PAD $\geq 90 \mathrm{~mm} \mathrm{Hg}$ ) fue del 55,8\%: cifras en torno al $50 \%$ y superiores se recogen en distintos trabajos de prevalencia de hipertensión arterial en el anciano en España y en el mundo (18-20). En relación con la edad y de forma paralela a lo demostrado por el estudio de Framingham (19), observamos una correlación significativa con la PAS de los sujetos estudiados. Aunque tam- bién detectamos el descenso de la PAD, éste sólo alcanzó significación estadística en el grupo de edad entre 71-75 años.

El porcentaje de individuos obesos (IMC $\geq 30 \mathrm{~kg} / \mathrm{m}^{2}$; $20,2 \%)$ es inferior al de otras series españolas $(21,22)$ y extranjeras $(23,24)$. Aunque se ha sugerido que la prevención del sobrepeso pudiera ser beneficiosa para mejorar el riesgo cardiovascular en las personas mayores de 65 años (25), tal como ocurre en la población de edad media, la obesidad no parece constituir un factor de riesgo para la mortalidad a 5 años en este grupo de edad (más bien, los riesgos asociados con una pérdida significativa de peso deberían ser motivo de un interés especial) (26).

También es menor que en otras series $(27,28)$ el porcentaje de consumo de tabaco en nuestro estudio $(10,1 \%$ en los varones; $1,9 \%$ en las mujeres). La gran diferencia por sexos observada refleja lo que es la constante del comportamiento de la población española en este grupo de edad (29). 


\begin{tabular}{|c|c|c|}
\hline $\begin{array}{l}\text { IMC } \geq 30 \mathrm{~kg} / \mathrm{m}^{2}(\mathrm{n}(\%)) \\
\text { PAS } \geq 140 \mathrm{~mm} \mathrm{Hg}(\mathrm{n}(\%)) \\
\text { PAD } \geq 90 \mathrm{~mm} \mathrm{Hg}(\mathrm{n}(\%)) \\
C T \geq 200 \mathrm{mg} / \mathrm{dl}(\mathrm{n}(\%)) \\
\operatorname{Tab}(\mathrm{n}(\%))\end{array}$ & $\begin{array}{l}13(19,9 \%) \\
33(50,8 \%) \\
14(21,5 \%) \\
49(75,4 \%) \\
3(4,6 \%)\end{array}$ & \\
\hline Al menos un factor de riesgo & $59(90,8 \%)$ & \\
\hline Sólo 1 factor de riesgo & $22(33,8 \%)$ & $\begin{array}{l}\text { PAS: } 4(6,2 \%) \text {; CT: } 17(26,2 \%) \text {; } \\
\text { Tab: } 1(1,5 \%) \text {. }\end{array}$ \\
\hline Sólo 2 factores de riesgo & $23(35,4 \%)$ & $\begin{array}{l}\text { IM C+PAS: } 1(1,5 \%) \text {; IM C+CT: } 6(9,2 \%) \text {; IM C+Tab: } 1 \\
(1,5 \%) \text {; PAS+PAD: } 3(4,6 \%) \text {; PAS+CT: } 11(16,9 \%) \text {; } \\
\text { PAD+CT: } 1(1,5 \%) \text {. }\end{array}$ \\
\hline Sólo 3 factores de riesgo & $13(20,0 \%)$ & $\begin{array}{l}\text { IM C+PAS+CT: } 4(6,2 \%) \\
\text { PAS+PAD+CT: } 9(13,8 \%) .\end{array}$ \\
\hline Sólo 4 factores de riesgo & $1(1,53 \%)$ & PAS+PAD+CT+Tab: $1(1,53 \%)$. \\
\hline Los 5 factores de riesgo & $0(0 \%)$ & IM C+PAS+PAD+CT+Tab: $0(0 \%)$ \\
\hline
\end{tabular}

IMC: índice de masa corporal (IM C: IMC $\geq 30 \mathrm{~kg} / \mathrm{m}^{2}$ ); PAS: Presión arterial sistólica (PAS: PAS $\geq 140 \mathrm{~mm} \mathrm{Hg}$ ); PAD: Presión arterial diastólica (PAD: PAD $\geq 90 \mathrm{~mm} \mathrm{HG}$ ); Tab: tabaquismo según los criterios recogidos en "M aterial y métodos" (Tab: Tab).

a Porcentajes calculados en relación con los 65 individuos de estudio que referían antecedentes personales de enfermedad cardiovascular.

Tiene especial relevancia el análisis de los resultados observados en el subgrupo de voluntarios ancianos de nuestro estudio con enfermedad cardiovascular previa. Aunque en ellos hay que suponer un seguimiento periódico por parte del sistema sanitario y un interés personal especial por sus problemas de salud (demostrado por la participación en estas jornadas), detectamos la presencia de al menos un factor de riesgo cardiovascular modificable (de entre los analizados) no controlado en el 90,8\% de los casos y niveles de CT por encima de lo deseable (>200 mg/dl) (8) en el 75\%. Este dato, y los que en el mismo sentido se recogen en la sección de resultados y en la tabla VI, refleja un deficiente control de la población anciana estudiada portadora de un riesgo cardiovascular elevado, lo cual hace suponer una situación similar, o aún peor, en la población general mayor de 65 años de nuestra provincia.

Campañas de prevención como las que aquí comentamos no sólo resultan útiles al despertar el interés de los habitantes de la zona por vigilar y conocer sus factores de riesgo, sino también al alertar a todos los niveles implicados en la asistencia sanitaria sobre alguna de sus deficiencias. Respecto al primer objetivo, durante las jornadas de Salamanca se comentó de forma individual con cada asistente la situación particular de riesgo cardiovascular de acuerdo con los parámetros analizados (y otros no tabulados en este trabajo, como los antecedentes personales de diabetes mellitus), indicando las medidas adecuadas para su control, la consulta con el equipo de aten- ción primaria o la evaluación por la Unidad de Lípidos del hospital, etc. Las jornadas incluyeron, además, varias "charlas-coloquio" sobre distintos aspectos, particularmente preventivos, de la arteriosclerosis. La labor de sensibilización social se completó con la participación de los organizadores en distintos medios de comunicación locales a través de los cuales, y con el pretexto de anunciar las jornadas, se emitieron mensajes de promoción de la salud cardiovascular.

Respecto al segundo objetivo (de alerta al sistema sanitario), el estado actual de las evidencias respecto a los factores de riesgo cardiovascular y los beneficios de la intervención en este grupo de edad se puede resumir en los siguientes puntos:

-Los perfiles de riesgo multivariado predicen con seguridad similar los incidentes cardiovasculares en los ancianos y en los jóvenes (30).

- La disminución de la morbi-mortalidad obtenida con el control de la hipertensión arterial ha sido claramente demostrada en los hipertensos mayores de 60 años (31-38) (aunque, hasta donde conocemos, no existen estudios que demuestren el beneficio del tratamiento hipotensor por encima de los 84 años).

—El abandono del hábito tabáquico se asocia a una reducción de la mortalidad, también a edades avanzadas $(39,40)$.

-En prevención primaria, la intervención farmacológica con lovastatina ha demostrado su eficacia en varones entre 45-73 años y en mujeres entre 55-73 años, con niveles medios de colesterol (estudio AFCAPS/TexCAPS) $(3,4)$. 
-En prevención secundaria, el tratamiento con simvastatina de pacientes hipercolesterolémicos, con antecedentes de cardiopatía isquémica, entre 64 - 70 años reduce significativamente la incidencia de eventos coronarios mayores y la mortalidad total y por cardiopatía isquémica (estudio 4S) (5); y la administración de pravastatina a enfermos normocolesterolémicos con antecedentes de infarto agudo de miocardio entre 60 - 75 años disminuye el número de eventos cardiovasculares y las muertes por cardiopatía isquémica (estudio CARE) (6).

(Llamamos la atención sobre el hecho de que las personas de más edad han sido, con frecuencia, excluidas de los ensayos clínicos más reconocidos en el área cardiovascular, por lo que no pueden beneficiarse -en uno $u$ otro sentido: intervencionista o abstencionista- de sus conclusiones).

\section{Bibliografía}

1. Instituto Nacional de Estadística. España en cifras 1999. http://www.ine.es/espcif/espcifes.htm.

2. Junta de Castilla y León. Conserjería de Economía y Hacienda. Dirección General de Asuntos Europeos. Castilla y León en la Unión Europea. http://www.jcyl.es/jcyl/ceh/dgae/svsaci/bde/Castilla-Leon-en-UE. htm.

3. Downs JR, , Clearfield M, Weis S, Whitney E, Shapiro DR, Beere PA. Primary prevention of acute coronary events with lovastatin in men and women with average cholesterol levels: results of AFCAPS/texCAPS. JAMA 1998; 279: 1615-1622.

4. Gotto AM Jr, Whitney E, Stein EA, et al. Relation between baseline and on-treatment lipid parameters and first acute major coronary events in the Air Force/Texas coronary atherosclerosis prevention study. Circulation 2000; 101: 477-84.

5. Miettinen TA, Pyörälä K, Olsson AG, et al. Cholesterol-lowering therapy in women and elderly patients with myocardial infarction or angina pectoris. Findings from the Scandinavian Simvastatin Survival Study (4S). Circulation 1997; 96: 4211-4218.

6. Sacks FM, Pfeffer MA, Moye LA, et al. The effect of pravastatatin on coronary events after myocardial infarction in patients with average cholesterol levels: Cholesterol and Recurrent Events (CARE). N Engl J Med 1996; 335: 1001- 1009.

7. Herrero Herrero JI, Martín Oterino JA, Sanz Ortega F, et al. Jornadas sobre colesterol y aterosclerosis en la provincia de Salamanca (1999): aspectos organizativos y análisis de factores de riesgo cardiovascular en la población estudiada. Clin Invest Arteriosclerosis 2000; 12: 83-88.

8. Sociedad Española de Arteriosclerosis, Sociedad Española de Medicina Interna y Liga de Lucha contra la Hipertensión arterial. Recomendaciones para la prevención primaria de la enfermedad cardiovascular. Clin Invest Arteriosclerosis 1994; 6: 62-102.

9. Fabiani F, Pérez Calero AM, Equipo colaborador de la Campaña de Prevención de la Hipercolesterolemia. Utilidad de las campañas para la prevención de la hipercolesterolemia. Clin Invest Arteriosclerosis 1998; 10: 296-298.

10. Aldea C, Puzo J, Pérez Lorenz JB, González Bonillo J, Olivera J, Ferrer M. Perfil lipídico en la población geriátrica de un medio rural de alta montaña. Clin Invest Arteriosclerosis 1998; 10: 291-295.

11. Ortega R, Pardo V, Martín V. Factores de riesgo de cardiopatía coronaria en el barrio de Santa Bárbara de Toledo. Hipertens Arterioscler 1991; 3: 16.

12. Cupples LA, D'Agostino RB. Some risk factors related to the annual incidence of cardiovascular disease and death using pooled repeated biennial measurements: Framingham study, 30-year follow up. En: Kannel WB, Wolf PA, Garrison RJ, editores. National Institutes of Health Publication No. 87-2703. Springfield, VA, US Department of Commerce, National Technical Information Service; 1997.

13. McNamara DJ. Dietary fatty acids and cholesterol. En: Archambaut P, Capron L, Detilleux M, editores. Cholesterol prevention primaire. París: Maison de la Chimie; 1990: p. 15-36.
Bajo la denominación de "ancianos" incluimos (con los criterios de edad tradicionales, seguramente precoces, adoptados en este trabajo) un grupo heterogéneo de sujetos en el que las intervenciones médicas (incluidas las farmacológicas) deben individualizarse considerando, entre otros factores además de la edad cronológica, la edad biológica y la expectativa de vida. Sin embargo, la aplicación a un individuo de este término genérico en la clínica diaria, puede inclinar a actitudes fatalistas, abstencionistas o tolerantes, que evidencias científicas como las mencionadas más arriba desaprueban. Es posible que esta tendencia se haya manifestado influyendo, de alguna forma, sobre los datos de nuestro estudio. En cualquier caso, éstos sugieren la necesidad de establecer estrategias para un mejor control del riesgo cardiovascular también en la población anciana de nuestra provincia.
14. Manolio TA, Furberg CD, Wahl PW. Eligibility for cholesterol referral in community-dwelling older adults. The Cardiovascular Health Study. Ann Intern Med 1992; 116: 641-649.

15. Kronmal RA, Cain KC, Zhan Y, et al. Total serum cholesterol levels and mortality risk as a function of age: a report based on the Framingham data. Arch Intern Med 1993; 153: 1065-1073.

16. Mattila KS, Marniemi J, Maki J, Juva K. Lipids, lipoproteins and apolipoproteins in the elderly. Scand J Clin Lab Invest 1996; 46: 131-136.

17. Nguyen PC, Hansson L, Petitet A, Safar ME. Blood pressure and serum lipids in hypertensive men and women aged 60-97 years. Blood Press; Suppl 3: 26-30.

18. Aranda P. Hipertensión arterial en el anciano. En: Aranda P, editor. Factores de riesgo vascular en el hipertenso. Madrid: Pharmaconsult; 1991: p. $457-460$.

19. Kannel WB, Vokonas PS. Cardiología preventiva en el anciano: el estudio de Framingham. En: Primer de cardiología preventiva. Dallas: American Heart Association; 1996: p. 194-203.

20. Joint National Commitee on Detection, Evaluation and Treatment of High Blood Pressure (JNC-V). Arch Intern Med 1993; 153: 154-183.

21. Sánchez García JI, Carratalá Pérez M, Molina Macía M, Server Romero L, Valencia Valencia P, Fernández Cañadas Sánchez JM. La magnitud de la gordura: prevalencia y tipo de obesidad en la población adscrita a un centro de salud. Aten Primaria 1998; 21: 159-164.

22. Gámez C, Ruiz-López MD, Artacho R, Puerta A, López MC. Body composition in institutionalized elderly people in Granada (Spain). Relation with other nutritional parameters. Int J Food Sci Nutr 1998; 49: 237-241.

23. Abrams J, Coultas DB, Malhotra D, Vela BS, Samaan SA, Roche RJ. Coronary risk factors and their modification: lipids, smoking, hypertension, estrogen, and the elderly. Curr Pob in Cardiol 1995; 20: 535610 .

24. Trenkwalder P, Ruland D, Lydtin H, Hense HW. Cardiovascular risk factors in patients over 65-years of age in Germany. Results of the STEPHY Study (English abstract). Z Kardiol 1994: 83: 803-809.

25. Harris TB, Savage PJ, Tell GS, Haan M, Kumanyika S, Lynch JC. Carrying the burden of cardiovascular risk in old age: associations of weight and weight change with prevalent cardiovascular disease, risk factors, and health status in the Cardiovascular Health Study. Am J Clin Nutr 1997; 66: 837-844.

26. Diehr P, Bild DE, Harris TB, Duxbury A, Siscovick D, Rossi M. Body mass index and mortality in nonsmoking older adults: the Cardiovascular Heatlth Study. Am J Public Health 1998; 88: 623-629.

27. Kannel WB. Epidemiology of cardiovascular disease in the elderly: an assessment of risk factors. Cardiovasc Clin 1992; 22: 9-22.

28. Moreiras O, Carvajal A, Beltrán B. Influencia del estado nutricional, juzgado por parámetros dietéticos y antropométricos, en la capacidad funcional de personas de edad avanzada. Seguimiento del estudio SENECA en España. Rev Gerontol 1995; 5: 353-360. 
29. Álvarez-Sala LA, González A, Millán J. El tabaco, un factor de riesgo cardiovascular evitable. En: Oya M, Garcés C, editores. Metabolismo lipídico. Sociedad y colesterol. Madrid: IDEPSA; 1997: p. 36-49.

30. Tabla de predicción de cardiopatía coronaria según los factores de riesgo. En: American Heart Association, editor. Primer de Cardiología preventiva. Dallas: American Heart Association; 1996: p. 226.

31. SHEP Cooperative Research Group. Prevention of stroke by antihypertensive drug treatment in older persons with isolate systolic hypertension. Final results of the Systolic Hypertension in the Elderly Program. JAMA 1991; 265: 3255-3266.

32. Bearden D, Allman R, McDonald R, Miller S, Pressel S, Petrovich H. Age, race, and gender variation in the utilization of coronary artery bypass surgery and angioplasty in SHEP. SHEP Cooperative Research Group. Systolic Hypertension in the Elderly Program. J Am Geriatr Soc 1994; 42: 1143-1149.

33. Amery A, Brixco P, Clement D, et al. Mortality and morbidity results from the European Working Party on High Blood Pressure in the Elderly Trial. Lancet 1985; 326: 1349-1354.
34. Report by the management committee: The Australian therapeutic trial in mild hipertension. Lancet 1980; 316: 1261-1267.

35. Dahlöf B, Lindholm LH, Hansson L, et al. Morbidity and mortality in the Swedish Trial in Old Patients with Hypertension (STOP-Hypertension). Lancet 1991; 338: 1281-1285.

36. Coope J, Warrender T. Ramdomized trial of treatment of hypertension in elderly patients in primary care. Lancet $1986 ; 328: 1145-1152$.

37. Medical Research Council Working Party. Medical Research Council trial of treatment of hypertension in older adults: principal results. BMJ 1992; 304: 405-412.

38. Staessen JA, Fagard R, Thijs L, et al. For the Systolic Hipertension in Europe Trial Investigators. Ramdomized double-blid comparison of placebo and active treatment for older patients with isolated systolic hypertension. Lancet 1997; 350: 757-764.

39. Hermanson B, Omen GS, Kronmal RA, et al. Beneficial six year outcome of smoking cessation in older men and women with coronary artery disease. N Eng J Med 1988; 319: 1365-1369.

40. Jajich CL, Ostfeld AM, Freeman DH, Smoking and coronary heart disease mortality in the elderly. JAMA 1984; 252: 2831-2834. 